UTHEP-291

December 1994

\title{
Finite Temperature Transition in Two Flavor QCD with Renormalization Group Improved Action
}

\author{
Y. Iwasaki ${ }^{\mathrm{a}}$, K. Kanaya ${ }^{\mathrm{a}}, \mathrm{S} . \mathrm{Sakai}^{\mathrm{b}}$ and T. Yoshiéa*

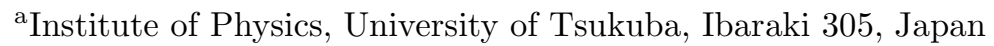

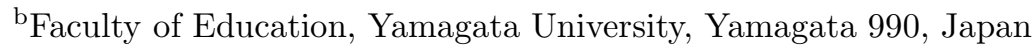

The finite temperature transition or crossover in QCD with two degenerate Wilson quarks is investigated using a renormalization group improved action. At $\beta=2.0$ and 2.1 where $a^{-1} \sim 1.0-1.2 \mathrm{GeV}$, the expectation value of the Polyakov loop and the pion screening mass on an $8^{3} \times 4$ lattice vary smoothly with the hopping parameter through the transition/crossover. The quark screening mass in the high temperature phase agrees well with that in the low temperature phase calculated on an $8^{4}$ lattice. The smooth transition of the observables is totally different from the sharp transition found for the standard action at $\beta=5.0$ and 5.1 where $a^{-1}$ is also $1.0-1.2$ $\mathrm{GeV}$.

\section{Introduction}

Recently MILC group has extensively investigated the finite temperature transition/crossover in the case of two degenerate Wilson fermions and found unexpected phenomena [1.2]. For $N_{t}=4$, when the quark mass is heavy the transition is smooth and when the quark mass is light it is also smooth. However, in the intermediate mass region $(\beta \sim 5.0)$, the transition is very sharp. This is completely opposite to what was supposed to be realized: It was supposed to become weaker or disappear. Furthermore, for $N_{t}=6$, in the range of intermediate mass, they observed clear two state signals.

We reproduce in fig.1 1 a the data[1] for the pion screening mass squared $m_{\pi}^{2}$, the expectation value of the Polyakov loop $L$ and the quark screening mass $m_{q}$ at $\beta=5.0$, together with our data [3]. The transition of the Polyakov loop is sharp and a cusp appears in the pion screening mass at the transition point. The quark mass defined through an axial-vector Ward identity 4 , peculiar behavior. The $m_{q}$ in the high temperature phase on the $N_{t}=4$ lattice behaves singularly and does not agree with that in the low temperature phase. This is in clear contrast with the fact that the $m_{q}$ is almost independent of whether

\footnotetext{
${ }^{*}$ Talk presented by T.Yoshié at Lattice 94 .
}

the system is in the high temperature phase or in the low temperature phase at $\beta=5.85$ in the quenched QCD [6] and at $\beta=5.5$ for $N_{f}=2$ case[7].

One possible reason for these phenomena is that we are far from the continuum limit. In this work, we study the finite temperature transition/crossover in the case of two degenerate quarks using a renormalization group (RG) improved action where we are supposed to be close to the continuum limit. Our main concern is to see whether the unexpected phenomena described above persist with the improved action.

\section{Improved action}

The RG improved action for pure gauge theory we proposed about ten years ago is

$S_{g}=1 / g^{2}\left\{c_{0} \sum\right.$ (plaquette $)+c_{1} \sum(1 \times 2$ loop $\left.)\right\}$ with $c_{1}=-0.331$ and $c_{0}=1-8 c_{1}$. Each oriented loop appears once in the sum. The form of the gauge action is determined by a block-spin RG analysis in the pure gauge theory [8]. We calculated the string tension [9, 10] and the hadron spectrum [1, 11] and investigated the topological properties and the $U(1)$ problem 12 in the quenched QCD with this improved action. We observe the following among others: The approach to the per- 

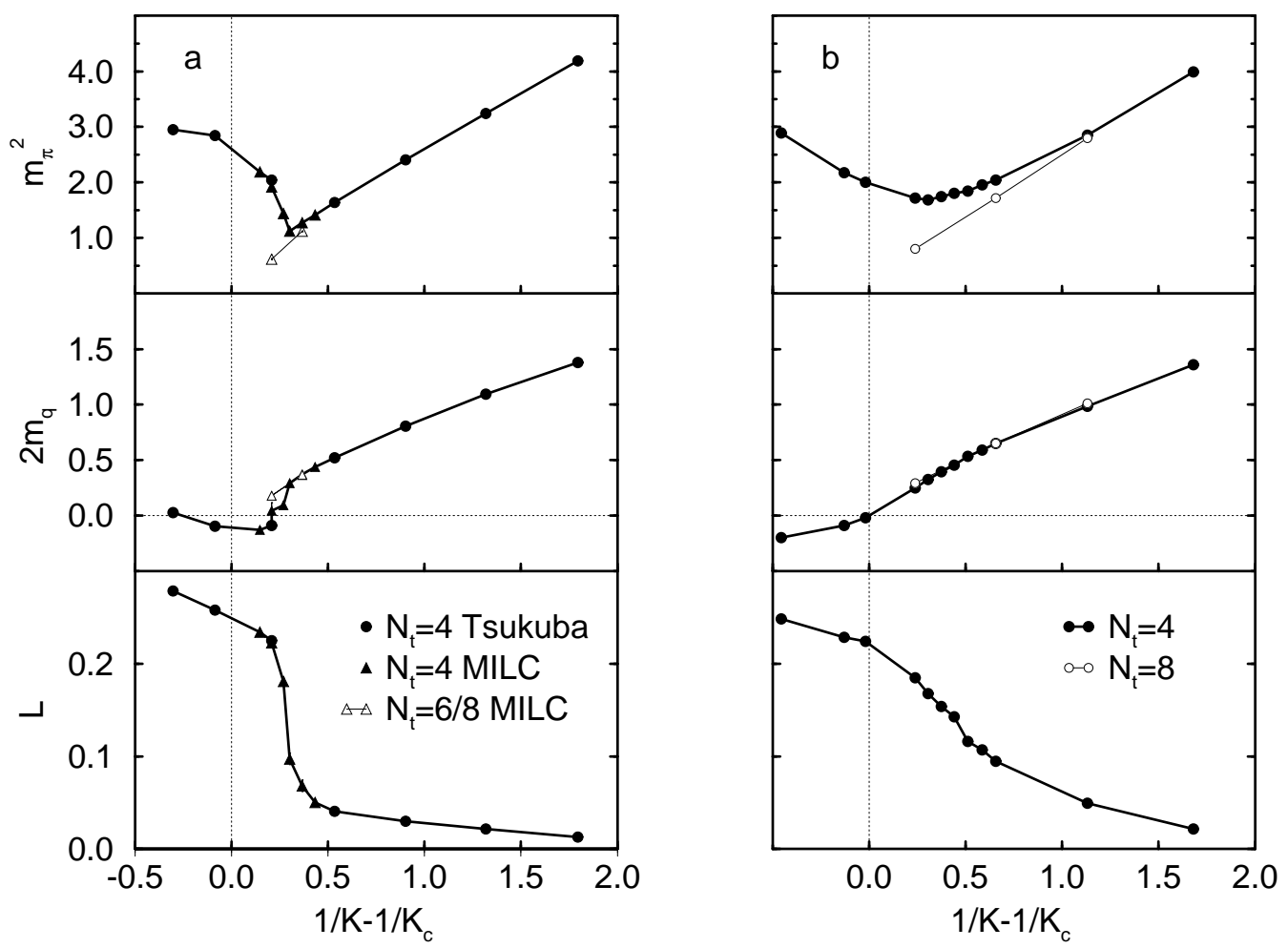

Figure 1. Observables on $N_{t}=4$ (filled symbols) and $N_{t}=6$ or 8 (open symbols) lattice. a) At $\beta=5.0$ with the standard action. Data are taken from [1] and [3]. b) At $\beta=2.0$ with the improved action.

turbative result for the expectation value of the plaquette is much faster than the case of the standard one-plaquette action [10]. Also note that the scale parameter of the improved action $\Lambda_{I M}$ is close to the $\Lambda_{\overline{M S}}: \Lambda_{\overline{M S}} / \Lambda_{I M} \sim 0.488[13$. Therefore the bare coupling of the improved action is already an improved coupling 14.

With these results it may be worthwhile to use a RG improved action for full QCD. The action we use as an improved action for full QCD is given by

$$
S=S_{\text {gauge }}^{I M}+S_{\text {quark }}^{\text {Wilson }} .
$$

The action is a sum of the RG improved action for gluons and the Wilson action for quarks. This action reduces to the improved pure gauge action in the heavy quark mass limit. There is also a possibility to improve the quark action. However we take the Wilson action for the quark action as a first step, because we believe that the ef- fect of the improvement of the gauge sector is much more significant than that of the quark sector by the following reasoning: After integration over fermion variables, the quark action may be written as a sum over various types of Wilson loops, assuming the convergence of hopping parameter expansion. The coefficients of various types of Wilson loops thus obtained are much smaller compared with $c_{1}=-0.331$ for the improved pure gauge action.

\section{Numerical Simulations}

Numerical simulations are done at $\beta=2.0$ and 2.1 on an $8^{3} \times 4$ lattice at several hopping parameters. In order to fix the scale and to compare the results in the high temperature phase with those in the low temperature phase, we also make simulations on an $8^{3} \times 8$ lattice. We use the 
hybrid Monte Carlo algorithm to generate gauge configurations with molecular dynamics step size $\Delta \tau=0.01$. Momentum refresh is done at every one unit of simulation time. Acceptance rates are larger than 0.92 for all cases. We discard first 100 $\sim 400$ trajectories for thermalization and use 100 $\sim 1000$ trajectories for measurement. The numbers of trajectories for thermalization and measurement are chosen taking into account whether the simulation point is close or not to the transition point. Wilson loops and Polyakov loops are measured at every trajectory. Screening correlation functions are measured at every 5 trajectories on an $8^{2} \times 16 \times N_{t}$ lattice obtained by doubling the original lattice.

Using the $\rho$ meson mass calculated on the $8^{3} \times 16$ lattice as an input, we obtain $a^{-1}=1.01$ $\mathrm{GeV}$ and $1.18 \mathrm{GeV}$ at $\beta=2.0$ and 2.1 , respectively. Therefore these $\beta$ 's correspond approximately to $\beta=5.0$ and 5.1 of the standard action, respectively.

\section{Results}

Fig.17b shows the results for the observables at $\beta=2.0$ versus $1 / K-1 / K_{c}$, where the $K_{c}$ is determined from the quark mass on the $N_{t}=4$ lattice. The data indicate the following: 1) The transition/crossover of the Polyakov loop around $1 / K-1 / K_{c} \sim 0.5$ is very smooth. 2) The hopping parameter dependence of the quark mass is also smooth and its value on the $N_{t}=4$ lattice agrees well with that on the $N_{t}=8$ lattice. This implies that the value of the quark mass is almost independent of whether the system is in the low temperature phase or in the high temperature phase. 3) The pion screening mass does not exhibit a cusp.

These features of the transition/crossover at $\beta=2.0$ are totally different from those observed for the standard action at $\beta=5.0$. Note that $a^{-1}$ are about $1 \mathrm{GeV}$ for both cases. We also observe a similar very clear contrast between the results at $\beta=2.1$ with the improved action and those at $\beta=5.1$ with the standard action where $a^{-1}$ are both approximately $1.2 \mathrm{GeV}$.

Furthermore preliminary results in the range of of $\beta=1.6-2.15$ indicate that the transitions are smooth from $\beta=2.15$ down to at least $\beta=1.8$. Therefore we conclude that the sharp transition in the intermediate mass region disappears with the improved action at $N_{t}=4$. This suggests a strong possibility that the first order phase transition observed by MILC at $N_{t}=6$ disappears with this action. To confirm this, we are planning to extend the work to a wider range of parameter, in particular, on a lattice with $N_{t}=6$.

The simulations are performed with Fujitsu VPP500/30 at University of Tsukuba. We would like to thank C. DeTar for providing us with data by MILC collaboration. This work is in part supported by the Grant-in-Aid of Ministry of Education, Science and Culture (No.06NP0601).

\section{REFERENCES}

1. C. Bernard et al., Phys. Rev. D49 (1994) 3574; Nucl. Phys. B (Proc. Suppl.) 34 (1994) 324.

2. C. Bernard et al., Phys. Rev. D46 (1992) 4741; T. Blum et al., ibid. D50 (1994) 3377.

3. Y. Iwasaki, K. Kanaya, S. Sakai and T. Yoshié, Nucl. Phys. B (Proc.Suppl.) 30 (1993) 327; ibid. 34 (1994) 314.

4. S. Itoh, Y. Iwasaki, Y. Oyanagi and T. Yoshié, Nucl. Phys. B274 (1986) 33.

5. M. Bochicchio et al., Nucl. Phys. B262 (1985) 331.

6. Y. Iwasaki, Y. Tsuboi and T. Yoshié, Phys. Lett. B220 (1989) 602.

7. Y. Iwasaki, K. Kanaya, S. Sakai and T. Yoshié, Phys. Rev. Lett. 67 (1991) 1494.

8. Y. Iwasaki, preprint UTHEP-118 (Dec. 1983) unpublished.

9. S. Itoh, Y. Iwasaki and T. Yoshié, Phys. Rev. D33 (1986) 1806.

10. S. Itoh, Y. Iwasaki and T. Yoshié, Phys. Lett. B185 (1987) 390.

11. S. Itoh, Y. Iwasaki and T. Yoshié, Phys. Lett. B183 (1987) 351; ibid. B167 (1986) 443.

12. S. Itoh, Y. Iwasaki and T. Yoshié, Phys. Lett. B184 (1987) 375; Phys. Rev. D36 (1987) 527.

13. Y. Iwasaki and S. Sakai, Nucl. Phys. B248 (1984) 441.

14. G. P. Lepage and P. B. Mackenzie, Phys. Rev. D48 (1993) 2250. 